\title{
Calcium-Independent Calcium/Calmodulin-Dependent Protein Kinase II in the Adult Drosophila CNS Enhances the Training of Pheromonal Cues
}

\author{
Jennifer E. Mehren and Leslie C. Griffith \\ Department of Biology and Volen Center for Complex Systems, Brandeis University, Waltham, Massachusetts 02454-9110
}

\begin{abstract}
Calcium/calmodulin-dependent protein kinase II (CaMKII) is abundant in the CNS and is crucial for cellular and behavioral plasticity. It is thought that the ability of CaMKII to autophosphorylate and become $\mathrm{Ca}^{2+}$ independent allows it to act as a molecular memory switch. We have shown previously that inhibition of Drosophila CaMKII leads to impaired performance in the courtship conditioning associative memory assay, but it was unknown whether the constitutive form of the kinase had a special role in learning. In this study, we use a tripartite transgenic system combining GAL4/UAS with the tetracycline-off system to spatially and temporally manipulate levels of $\mathrm{Ca}^{2+}$-independent CaMKII activity in Drosophila. We find an enhancement of information processing during the training period with $\mathrm{Ca}^{2+}$-independent, but not $\mathrm{Ca}^{2+}$-dependent, CaMKII. During training, control animals have a lag before active suppression of courtship begins. Animals expressing $\mathrm{Ca}^{2+}$-independent CaMKII have no lag, implying that there is a threshold level of $\mathrm{Ca}^{2+}$-independent activity that must be present to suppress courtship. This is the first demonstration, in any organism, of enhanced behavioral plasticity with overexpression of constitutively active CaMKII. Anatomical studies indicate that transgene expression in antennal lobes and extrinsic mushroom body neurons drives this behavioral enhancement. Interestingly, immediate memory was unaffected by expression of T287D CaMKII in mushroom bodies, although previous studies have shown that CaMKII activity is required in this brain region for memory formation. These results suggest that the biochemical mechanisms of CaMKII-dependent memory formation are threshold based in only a subset of neurons.
\end{abstract}

Key words: CaMKII; courtship conditioning; Drosophila, tetracycline; constitutive kinase; GAL4/UAS

\section{Introduction}

Decreased calcium/calmodulin-dependent protein kinase II (CaMKII) activity is associated with impaired learning, memory, and cellular plasticity in a variety of organisms. In Drosophila, animals expressing a CaMKII inhibitor, ala peptide, are unable to form memory in an associative learning assay called courtship conditioning (Griffith et al., 1993). This assay manipulates a naturally occurring interaction between males and females. Male flies trained with a mated female reduce courtship during training in response to aversive cues given off by the mated female. After training, the male courts a virgin female at a significantly lower level than would a naive male (Siegel and Hall, 1979). Courtship suppression reflects associative memory, requiring both attractive and aversive pheromonal cues (for review, see Greenspan, 1995; Hall, 2002).

Received Aug. 30, 2004; revised 0ct. 13, 2004; accepted 0ct. 15, 2004.

This work was supported by National Institutes of Health Grants P01 NS44232 (L.C.G.) and F32 NS43024 (J.E.M.). We thank A. Villella, J. Hall, P. Sengupta, J. Levine, and J. J. L. Hodge for helpful discussions and comments on this manuscript, M. Rosbash for use of the lumenometer, B. Bello for tTA and tet0 transgenic flies, and E. Dougherty (Brandeis Confocal Facility, Waltham, MA; supported by National of Institutes of Health Grants P30 NSO45713 and S10 RR16780) and S. Karel for technical assistance.

Correspondence should be addressed to Leslie C. Griffith, Department of Biology, MS008, Brandeis University, 415 South Street, Waltham, MA 02454-9110. E-mail: griffith@brandeis.edu.

DOI:10.1523/JNEUROSCI.3560-04.2004

Copyright $\odot 2004$ Society for Neuroscience ～0270-6474/04/2410584-10\$15.00/0
Demonstrating that a behavior requires CaMKII activity only scratches the surface of the role of CaMKII. CaMKII can exist in several activity states that depend on autophosphorylation. In its basal state, without autophosphorylation, the enzyme is tightly regulated by $\mathrm{Ca}^{2+} / \mathrm{CaM}$, being inactive in its absence. When the enzyme is autophosphorylated at T286 (or T287 for Drosophila CaMKII), it becomes independent of $\mathrm{Ca}^{2+} / \mathrm{CaM}$ and can have as much as $80 \%$ of its maximal $\mathrm{Ca}^{2+}$-stimulated activity in the absence of $\mathrm{Ca}^{2+} / \mathrm{CaM}$ (for a review of CaMKII biochemistry, see Hudmon and Schulman, 2002). The switch between $\mathrm{Ca}^{2+}$ dependent and $\mathrm{Ca}^{2+}$-independent activity has been proposed to be an important early event in synaptic plasticity in a number of systems (Lisman et al., 2002). A behavioral requirement for activity does not address whether the formation of $\mathrm{Ca}^{2+}$. independent activity is important.

When T287 of Drosophila CaMKII or T286 of mammalian $\alpha$ CaMKII is mutated to aspartate (T287D for fly kinase), mimicking the autophosphorylated form of the enzyme, the kinase becomes constitutively active (Fong et al., 1989; Waldmann et al., 1990; Wang et al., 1998). Mutation of T286/7 to alanine (T287A for fly kinase) results in a kinase that has normal $\mathrm{Ca}^{2+}$-dependent activity but can never become independent of this regulator. These kinase mutants can be used to probe the role of $\mathrm{Ca}^{2+}$ independence in cellular processes. Previous work in Drosophila suggests that the two forms of CaMKII have different cellular 
roles; neural expression of T287D CaMKII throughout development caused lethality, morphological defects, and decreased neuronal excitability, whereas expression of T287A CaMKII had no consequences (Park et al., 2002). These studies are consistent with constitutively active CaMKII having a unique function in some populations of neurons that cannot be duplicated by the $\mathrm{Ca}^{2+}$-dependent form of the kinase. In rodents, overexpression of T286D $\alpha$ CaMKII impairs both spatial and contextual memory (Bach et al., 1995; Mayford et al., 1996; Bejar et al., 2002) rather than enhancing the process, as might be expected from the simple "molecular switch" model. This failure to enhance behavior in mammals may be attributable to saturation of plasticity mechanisms (Lledo et al., 1995; Bejar et al., 2002). No overexpression studies using the $\mathrm{Ca}^{2+}$-dependent form of CaMKII (T286A) have been done in rodents.

To test the roles of CaMKII activity states in learning and memory, we used the GAL4/UAS system to express T287A CaMKII, and we combined the GAL4/UAS system with the tetoff system to express T287D in multiple areas of the adult nervous system known to require CaMKII for associative memory formation. These studies provide the first behavioral evidence that constitutively active CaMKII can enhance plasticity and demonstrate that CaMKII has multiple biochemical mechanisms for modulating memory formation.

\section{Materials and Methods}

Fly strains and crosses. Fly stocks and crosses were maintained on cornmeal, yeast, dextrose, and agar medium and kept at $18^{\circ} \mathrm{C}$ or $25^{\circ} \mathrm{C}$ in a 12 $\mathrm{hr}$ light/dark cycle. Tetracycline (tc) feeding was performed by growing flies on medium containing tetracycline at $10 \mu \mathrm{g} / \mathrm{ml}$ food. Each bottle contained $\sim 75 \mathrm{ml}$ of food, and each vial contained $\sim 8 \mathrm{ml}$ of food. tc was added and mixed into the food after cooking but before solidification when the food temperature was $55^{\circ} \mathrm{C}$ or below. For each liter of tetracycline-containing food, $2 \mathrm{ml}$ of tc $(5 \mathrm{mg} / \mathrm{ml}$, dissolved in $95 \%$ EtOH) was added. Tetracycline concentrations of as little as $0.1 \mu \mathrm{g} / \mathrm{ml}$ food have been shown to inactivate tTA (tetracycline-controlled transactivator protein) when combined with tetO-lacZ (Bello et al., 1998). With our system, however, we detected a low level of GFP (green fluorescent protein) in third-instar larvae carrying one copy each of pan-neuronal MJ85b-GAL4 (Joiner and Griffith, 1997), UAS-tTA, and tetO-GFP grown on food containing tc at $5 \mu \mathrm{g} / \mathrm{ml}$ food (data not shown). Full suppression of tetO-GFP expression was seen with tetracycline at $10 \mu \mathrm{g} / \mathrm{ml}$ food, and therefore this was the concentration used for all experiments described in this paper. Suppression of tet $O$ gene expression was obtained in adults by daily feeding $100 \mu \mathrm{g} / \mathrm{ml}$ tc in a $4 \%$ sucrose solution.

When crosses were performed for tc feeding, the mothers of the parental cross were taken from stocks maintained on tetracycline food for more than two generations. Canton $S$ males were used as the wild type, and all transgenic lines were in a Canton S, white $(w)$ genetic background. $U A S-t T A^{220}, U A S-t T A^{221}$, and all tetO-luciferase (luc) and tetO-GFP lines were gifts from Bruno Bello (University of Basel, Basel, Switzerland). $U A S-t T A^{29}$, which has less background expression, was produced by "jumping" the tetO-luc P-element from line UAS- $t T A^{221}$ with $\Delta 2-3$ transposase. Virgin females homozygous for $U A S-t T A^{221}$ were crossed to w; TM2, Ubx $\Delta 2-3 / \mathrm{MKRS}, \mathrm{Sb} \Delta 2-3$ males. From this cross, $S b$, coloredeyed, male progeny were collected and crossed to virgin females of the genotype w; Sp/CyO; Sb/TM6B. From this second cross, colored-eyed, $\mathrm{Cy}, \mathrm{Hu}$, non-Sb males were collected and individually crossed to Canton $\mathrm{S}, w$ virgin females to determine in which chromosome the P-element was located. Finally, progeny with colored eyes were crossed to each other to produce homozygotes. The GAL4 lines MJ85b and MJ94 were generated by Mei-Ling Joiner (University of Iowa, Iowa City, IA) (Joiner and Griffith, 1997, 1999). The other GAL4 lines used have been described previously and are C155 (Lin and Goodman, 1994), 29BD (Joiner and Griffith, 1999), 30Y (Yang et al., 1995), and 201Y (Yang et al., 1995). The tetO-CaMKII-T287D flies were made by cloning mutated CaMKII sequence into the WTP2 vector (Bello et al., 1998).
Luciferase assay. Flies carrying one copy each of UAS-tTA and tetO-luc (virgin Canton $\mathrm{S}$ females were crossed to double-homozygous UAS-tTA; tetO-luc males) and flies carrying one copy each of MJ85b-GAL4, UAS$t T A$, and tetO-luc (virgin MJ85b females were crossed to doublehomozygous UAS-tTA; tetO-luc males) were grown either on or off tc medium. Newly-eclosed males were collected and processed as described below, either immediately or after being grouped in food vials for 1-5 d. Protein was extracted from six male heads per sample by homogenizing in $50 \mu \mathrm{l}$ of PBS. The homogenized samples were centrifuged for $2 \mathrm{~min}$, and $40 \mu \mathrm{l}$ of supernatant per sample was collected. Bright-Glo luciferase assay reagent ( $20 \mu \mathrm{l}$; Promega, Madison, WI) was added to each sample; after a $3 \mathrm{~min}$ incubation period at room temperature, each sample was read on a TD-20/20 DLReady luminometer (Turner Biosystems, Sunnyvale, CA). Results are expressed as the amount of lumens emitted from the tetracycline-fed MJ85b; UAS-tTA/+; tetO-luc/+ fly heads, as a percentage of the lumens emitted from male fly heads of the same genotype, which were not maintained on tetracycline. To control for background, lumen measurements of control heads of the genotype UAS-tTA/+; tet $O-l u c /+$ (the homozygous UAS- $t T A^{29}$; tet $O-l u c^{8 B}$ line out-crossed to Canton S) maintained on tc or not, were subtracted from their appropriately matched experimental samples before reaction-product calculations.

Anatomy. Brains and chemosensory organs (antennae, proboscises, and forelegs) were dissected in PBS from 5- to 6-d-old adult male flies. Brains were fixed in 4\% paraformaldehyde for $20-30 \mathrm{~min}$, washed three times in PBS, and mounted in glycerol. Chemosensory organs were either fixed for $20 \mathrm{~min}$ or directly mounted in glycerol. Images were acquired using the $20 \times$ (forelegs and proboscises) or $40 \times$ (brains and antennae) objective on a Leica (Nussloch, Germany) TCS SP2 confocal scanning microscope.

Behavior. All behavioral observations were performed at $25^{\circ} \mathrm{C}$ and $70 \%$ relative humidity in a Harris Environmental Room. To eliminate visual stimuli, flies were tested in dim red-light conditions. Fly pairs were placed in Plexiglas mating chambers ( $8 \mathrm{~mm}$ diameter, $3 \mathrm{~mm}$ high). To prevent dehydration, and a circle of filter paper soaked in distilled water was placed at the bottom of each courtship chamber. On the day before conditioning, wild-type females were fertilized by wild-type males and then grouped in a food vial until the next day. For courtship conditioning, one 4- to 6-d-old male was placed with a 3- to 5-d-old wild-type female that had mated the day before. After $1 \mathrm{hr}$ of training, the male was transferred to a new chamber and observed for a 10 min test period with a $\mathrm{CO}_{2}$-anesthetized virgin female collected that day. Sham controls involved placing a male alone in a chamber for the duration of the training and then testing as above.

The first and last $10 \mathrm{~min}$ of the $1 \mathrm{hr}$ training period, and the $10 \mathrm{~min}$ test and sham test periods, were videotaped. For each of the $10 \mathrm{~min}$ observation periods recorded, a courtship index (CI) was measured for each male tested. CI is defined as the amount of time the male spent courting during the observation period. Test males who either copulated during training or who had initial CIs (during the first $10 \mathrm{~min}$ of training) of $<0.10$ were not used in analysis. If an anesthetized virgin awakened or the fly pair copulated during the test, the observation period was terminated. Training indices were calculated by dividing the $\mathrm{CI}$ for the final 10 min period of the conditioning period $\left(\mathrm{CI}_{\mathrm{f}}\right)$ by that for the initial $10 \mathrm{~min}$ period $\left(\mathrm{CI}_{\mathrm{i}}\right)$. Memory was calculated by taking the $\mathrm{CI}$ of the $10 \mathrm{~min}$ test period $\left(\mathrm{CI}_{\mathrm{t}}\right)$ as a fraction of the mean sham index $\left(\mathrm{mCI}_{\mathrm{sh}}\right)$ for the various treatments. A memory index of 1.0 means no memory was formed; a memory index $<1.0$ indicates memory.

Habituation to immature males was assayed by pairing a male with either an immature male (collected that day) or for the control, a mature male. The mature male pairing control was done to eliminate the possibility of fatigue accounting for reduced courtship after training. Exposure to a mature male should cause no habituation to immature males. A $\mathrm{CI}$ for the first and final $10 \mathrm{~min}$ of the training period was measured, and a training index $\left(\mathrm{CI}_{\mathrm{f}} / \mathrm{CI}_{\mathrm{i}}\right)$ was calculated. Immediately after training or control training, males were tested with a new immature male. A CI was calculated for the 10 min test period, and a habituation index $\left(\mathrm{CI}_{\mathrm{t}} / \mathrm{mCI}_{\text {con }}\right)$ consisting of the ratio of the test $\mathrm{CI}$ for the trained male over the $\mathrm{CI}$ for the control trained males was calculated. A habituation index of 1.0 means no habituation has occurred; a habituation index $<1.0$ indicates habituation. 
To control for general sluggishness attributable to nonspecific effects of the multiple transgenes, locomotion was measured by counting the number of times a male crossed a diameter across the mating chamber in $3 \mathrm{~min}$ (see supplemental Table 1, available at www.jneurosci.org as supplemental material). Locomotion measurements were performed separately from the courtship conditioning trials. Hemizygous MJ85b and heterozygous $201 Y$ and $30 Y$ males were slightly less active than wild-type males. However, flies of these genotypes courted females normally (data not shown), indicating that this hypoactivity did not account for the changes in courtship plasticity we observed.

Statistics. Each CI was subjected to arcsine, arcsine squared, or arcsine square root transformation to effect approximation of normal distributions (Villella and Hall, 1996; Joiner and Griffith, 1997; Villella et al., 1997). Transformed learning or memory scores were subjected to a one-way ANOVA with genotype as the main effect, using JMP (version 5.0 for Macintosh; SAS Institute, Cary, NC), and planned comparisons of means were performed with Student's $t$ test (adjusted for experimentwise error) (Sokal and Rohlf, 1995).

Locomotor measurements were normally distributed, and raw data were subjected to a one-way ANOVA with genotype as the main effect, using Statview (version 4.5 for Macintosh; SAS Institute). Statistical results are summarized in the legends to Figures 3, 4, supplemental Figure 1, and supplemental Table 1 (available at www.jneurosci.org as supplemental material).

\section{Results}

Calibration of the

\section{GAL4/UAS/tet-off system}

The early lethality of T287D CaMKII when expressed under control of neuronal GAL4 lines (Park et al., 2002) necessitated that we control expression of this transgene temporally. To be able to use the wide array of GAL4 lines already known to express in neurons critical to courtship conditioning, we developed a tripartite expression system that melds the spatial control inherent to GAL4 enhancer traps (Brand and Dormand, 1995) with the temporal control that can be achieved using tet-off (Bello et al., 1998; Blau and Rossi, 1999). To use this system in Drosophila, three elements are required: a GAL4 transgene, a tTA fused downstream of UAS, and the gene of interest downstream of a tet $O$ promoter sequence (Fig. $1 A$ ). In such animals grown on tetracycline-containing food, tTA is unable to bind to the tet $O$ promoter, and tet $O$ gene expression is kept off. If the fly is not under the influence of tetracycline, it expresses the tet $O$ gene in the same spatial pattern as the GAL4 protein.

To confirm that this system works in the nervous system of Drosophila, we used the pan-neuronal GAL4 line MJ85b to drive expression of tetO-GFP. We found that tc, when mixed into the larval medium at a concentration of $10 \mu \mathrm{g} / \mathrm{ml}$, repressed GFP expression in larvae and pupae carrying one copy each of MJ85bGAL4, UAS-tTA, and tetO-GFP (Fig. $1 B$ ), whereas $5 \mu \mathrm{g} / \mathrm{ml}$ did not (data not shown). Because feeding flies tc in the larval medium at a concentration of $10 \mu \mathrm{g} / \mathrm{ml}$ was sufficient to inhibit tTA activity through pupal development, this concentration was used in all of the behavioral experiments in this study.
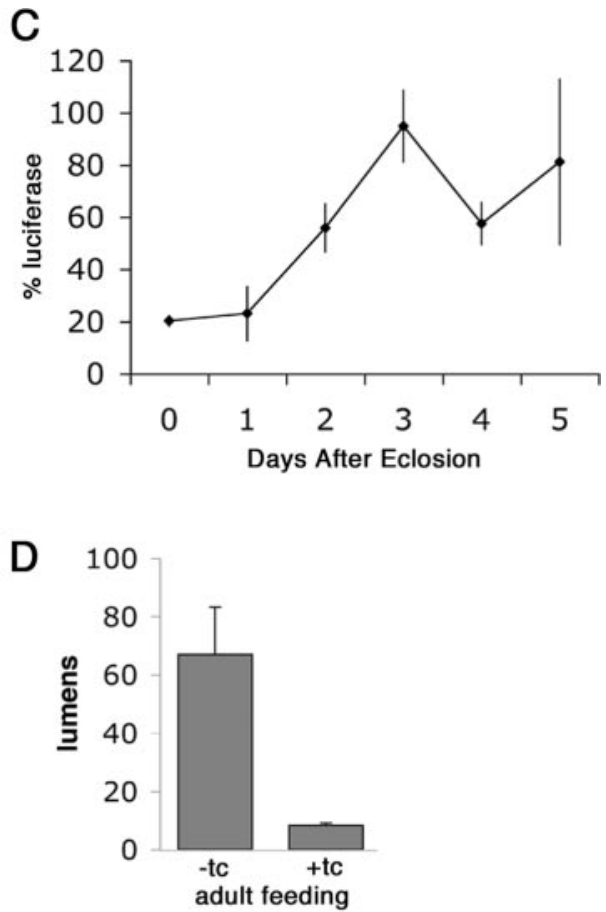

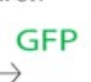

adult feeding

Figure 1. The GAL4/UAS/tet-off system. $A$, Schematic of transgenes used. When a fly carrying a GAL4 driver is crossed to a fly with UAS-tTA and tetO gene constructs (GFP is shown here as an example), the resulting progeny are triply transgenic. When in the larval medium at $10 \mu \mathrm{g} / \mathrm{ml}$, tTA is unable to activate the tetO promoter, and the tetO gene is kept silent. Shown are grown at $25^{\circ} \mathrm{C}$. Each sample consists of protein from six heads; $n=3$ for each time point. $D$, Feeding $\mathrm{tc}(100 \mu \mathrm{g} / \mathrm{ml}$ in a $4 \%$ sucrose solution) to MJ85b; UAS-tTA ${ }^{29} /+$; tet0-luc $8 B /+$ adults for $5 \mathrm{~d}$ after eclosion suppresses tet0-luciferase expression.

Time course of gene induction after tetracycline removal

How long does it take for inhibition of the tet $O$ gene to be reversed after removal of tetracycline from the food? We determined a time course of induction by using a luciferase reporter under the control of a tetO promoter. MJ85b-GAL4 virgin females were crossed to UAS-tTA; tetO-luc males in vials containing either tc-free or tc-laced medium. Figure $1 C$ shows the amount of luciferase expression in heads from male progeny (MJ85b; UAS-tTA/+; tetO-lucl+) grown on tc, as a percentage of heads of the same genotype grown on tc-free food. Background tetO-luc expression was determined by crossing wild-type females to UAS-tTA; tetO-luc males, in medium with or without tc, and measuring the luciferase levels of male heads in parallel with the GAL4 crosses.

Tetracycline-fed flies were collected within $2 \mathrm{hr}$ of eclosion and then either processed immediately for luciferase measurement or aged in vials containing tc-free food before luciferase measurement. In newly eclosed heads of triple-transgenic flies fed regular (tc-free) food, the average measurement of luciferase expression was $586.2 \pm 6.9$ lumens/homogenate (mean \pm SEM; six heads per homogenate; $n=3$ ), whereas regularly fed (tc-free) flies that lacked the GAL4 element emitted 133.2 \pm 5.5 lumens/ sample. In newly eclosed heads of tc-fed, triple-transgenic flies, an average of $143.2 \pm 11.1$ lumens were emitted per six-head sample, whereas the tc-fed flies without GAL4 emitted an average of $51.8 \pm 2.3$ lumens per six heads. These results indicate that tc 
Table 1. Tetracycline feeding rescues toxic effects of overexpression of activated CaMKII

\begin{tabular}{lllll}
\hline GAL4 & UAS-tTA & tet0-T287D & -tcphenotype & +tcrescue \\
\hline C155 & 220 & 7.1 & Lethal & Yes \\
C155 & 220 & 9.3 & Lethal & Yes \\
29BD & 220 & 7.1 & Lethal & Yes \\
29BD & 220 & 9.3 & Lethal & Yes \\
29BD & 221 & 3.4 & Infantile & Yes \\
29BD & 29 & 3.4 & Infantile & Yes \\
MJ85b & 220 & 7.1 & Lethal & Yes \\
MJ85b & 221 & 3.4 & Small & Yes \\
MJ85b & 221 & 3.3 & Small & Yes \\
MJ85b & 29 & 3.4 & Small & Yes
\end{tabular}

Lethality of T287D CaMKII is rescued by tetracycline suppression of expression during development. Females of each GAL4 line were crossed to flies carrying UAS-tTA and tetO-T287D transgenes and grown with or without tetracycline in the larval medium. Adult progeny were counted, and the percentage viability and/or display of infantile characteristics of triple-transgenic progeny was recorded. All tetracycline-fed crosses completely rescued lethality or infantile characteristics, producing flies with wild type-like appearance. Data are shown for three different GAL4 lines, three different UAS-tTA insertions, and four different tet0-T287D insertions. - tc phenotype, Flies crossed without tc medium; $+t$ tc rescue, flies crossed with tc medium.

inhibits tTA function through pupal development, but that both the UAS-tTA and tetO-luc constructs have "leaky" expression that is manifested by luciferase expression in the absence of GAL4. "Leakiness" is a common feature of transgenes and is believed to be a function of the insertion site of the individual transgene. Insertion site can also influence the level of expression of inducible transgenes (see below).

We can determine the dynamics of GAL4-induced tTA activation of the $t e t O$ reporter by looking at luciferase activity as a function of time on tc-free food and subtracting background expression levels. When grown at $25^{\circ} \mathrm{C}$ through development, by $5 \mathrm{~d}$ after eclosion (the age at which behavioral experiments are done), luciferase expression in tetracycline-fed MJ85b; UAS$t T A /+; t e t O-l u c /+$ flies increased to $81 \%$ that of flies of the same genotype that were never exposed to tc (Fig. 1C). At $8 \mathrm{~d}$ after eclosion, luciferase expression was still at $\sim 80 \%$ (data not shown). These results show that the tetO-luc expression in tc-fed triple-transgenic flies is induced by GAL4 activation of tTA after removal of tetracycline. The tetO-luc induction in tc-fed, MJ85b; $U A S-t T A /+$; tetO-luc/ + flies levels off at a value below the maximal level of that obtained in regularly fed flies of the same genotype; this antibiotic-feeding ceiling effect has also been seen in mice (Bejar et al., 2002). We were able to obtain suppression of transgene expression in adulthood by feeding adults tc-laden sucrose solution. Figure $1 D$ shows luciferase activity in 5-d-old flies removed from tc at eclosion or fed tc in sucrose. Flies fed as adults had much lower levels of luciferase activity $\left(P_{(1,4)}=0.0694\right.$; Student's $t$ test).

\section{Tetracycline rescues toxic developmental effects of}

\section{$\mathrm{Ca}^{2+}$-independent T287D CaMKII}

Park et al. (2002) reported reduced viability as well as underinflated wings and abdomens ("infantile" phenotype) in adult flies expressing the T287D form of CaMKII in the nervous system throughout development. We replicated these adult abnormalities by expressing tet $O-T 287 \mathrm{D}$ under the control of neuronal GAL4 drivers and UAS- $t T A$ in the absence of tc. Table 1 displays the results of crossing GAL4 lines MJ85b, 29BD, or C155 to UAS$t T A$; tetO-T287D flies, with or without tetracycline in the larval medium. MJ85b expresses GAL4 throughout the CNS and PNS (Joiner and Griffith, 1997). The C155-GAL4 transgene is inserted near elav and expresses in all neurons, beginning during embryogenesis (Lin et al., 1994). 29BD-GAL4 expresses in the $\alpha$-, $\beta$-,

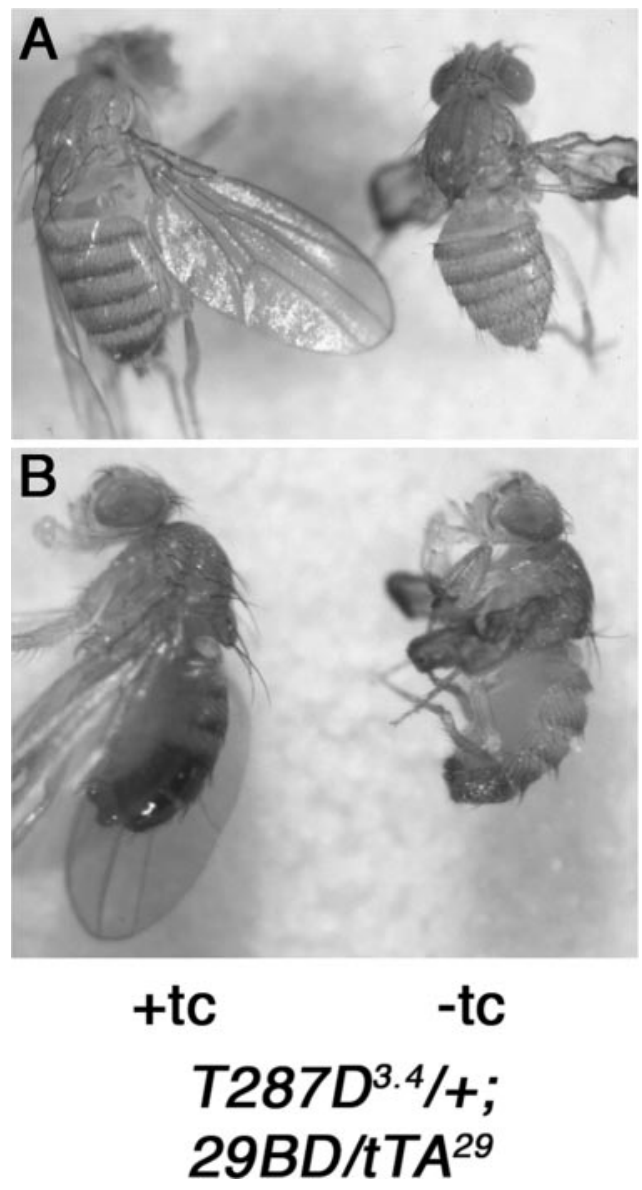

Figure 2. Tetracycline feeding rescues T287D-induced morphological defects. Female $(A)$ and male ( $B$ ) flies of the genotype UAS-tTA $A^{29} /+; 29 B D /$ tet0-T287D $D^{3.4}$ are shown. Flies on left side of each panel were grown on $10 \mu \mathrm{g} / \mathrm{ml}$ tetracycline. Flies on the right side were grown on normal, tetracycline-free food.

and $\gamma$-lobes of the mushroom bodies, as well as groups of cells in the mediolateral protocerebrum and dorsolateral subesophageal ganglion (Joiner and Griffith, 1999). Flies expressing the tetO$T 287 D^{7.1}$ transgene driven by $29 B D, M J 85 b$, or C155 were not viable when raised on tetracycline-free food. However, they were viable and similar to wild-type flies in appearance when fed tetracycline in the larval medium. The same was true for flies expressing tetO-T287D ${ }^{9.3}$ in the $29 B D$ or $C 155$ pattern. With transgene insertions tetO-T287D $\mathrm{D}^{3.3}$ and tetO-T287D $\mathrm{D}^{3.4}, \mathrm{MJ} 85 \mathrm{~b}$ driving tet $O-T 287 D$ resulted in viable, but much smaller, adults. In the case of tetO-T287D ${ }^{3.4}, 29 \mathrm{BD}$-driven expression caused a fully infantile adult phenotype, including hyper-contracted bodies and furled wings. The difference in phenotype produced by these independent tetO-T287D transgenes is likely to be attributable to difference in their genomic insertion site. As shown in Table 1 and Figure 2, tc feeding rescues viability and adult morphological phenotypes produced by all of the transgenes.

The rescue of lethal or morphological phenotypes by adding tetracycline to the larval medium illustrates three points. (1) The phenotypes we observe are a direct result of tet $O-T 287 D$ expression. (2) The tc dosage of $10 \mu \mathrm{g} / \mathrm{ml}$ food during larval life inhibits the tetracycline-transactivator protein through metamorphosis. (3) The background level of leaky expression of the transgenes in the presence of tetracycline is insufficient to cause developmental abnormalities. 


\section{Adult T287D CaMKII expression enhances plasticity during training}

To determine whether expression of constitutively active CaMKII had effects on learning and memory, we used the courtship conditioning assay. Mated females are believed to emit both stimulatory and aversive pheromonal cues. The association of these cues during training causes the male to suppress subsequent courtship (Siegel and Hall, 1979; Tompkins et al., 1983). Males were trained with fertilized, wild-type females and tested for short-term memory immediately after training with a newly eclosed anesthetized wild-type virgin (as described by Joiner and Griffith, 1997, 1999, 2000). For each genotype tested, shamtrained males (who spent the $1 \mathrm{hr}$ training period alone in a courtship chamber) were also tested with anesthetized virgins (see Materials and Methods).

The courtship conditioning paradigm has been shown to have two distinct behavioral outputs. During the training period, the male will modify his courtship of the mated female. This behavior requires CaMKII activity in antennal lobes and parts of the lateral protocerebrum (Joiner and Griffith, 1999). It is not known whether this suppression is associative or non-associative. During the memory assay with the virgin tester, the suppression of courtship depends on CaMKII activity in mushroom bodies, central complex, and distinct areas of the lateral protocerebrum (Joiner and Griffith, 1999). Courtship suppression during the test phase is known to be associative (Tompkins et al., 1983). These two behavioral outputs use different neuronal circuits and can be independently modified (Kane et al., 1997; Joiner and Griffith, 2000).

Assessment of the level of courtship during training and testing was done by calculating a CI. CI is the fraction of time spent courting during an observation period. Learning during the training period was measured as the ratio of the CI of the final 10 $\min \left(\mathrm{CI}_{\mathrm{f}}\right)$ to the initial $10 \mathrm{~min}\left(\mathrm{CI}_{\mathrm{i}}\right)$. Training indices (Joiner and Griffith, 1997, 1999, 2000) of $<1.0$ indicate a decrease in courtship of the mated female during the training period; the smaller the value of the training index, the better the learning.

To control for background effects of the multiple P-element insertions, we tested male progeny of wild-type females crossed to the UAS-tTA; tetO-T287D lines (Fig. 3), and male progeny of GAL4 virgin females crossed to wild-type males (supplemental Fig. 1, available at www.jneurosci.org as supplemental material). Wild-type males maintained on tc medium for two or more generations were also tested as controls. Wild-type males raised on tc food showed a mean training index of $0.55 \pm 0.12$, which is similar to what we have seen for wild-type flies maintained on regular food $\left(0.37 \pm 0.16 ; t\right.$ test; $\left.P_{(1,42)}=0.3095 ; \alpha=0.05\right)$. Therefore, tc feeding does not adversely affect learning ability during the training period. Males with one copy of each of the effector transgene constructs, tetO-T287D/+; UAS-tTA/+, showed similar performance compared with wild type $(t$ test comparing the effector line with tc-fed wild type, with $\alpha$ level of $\left.0.05 ; P_{(1,39)}=0.1635\right)$, indicating that leakiness of the tet $O$ $T 287 D$ or UAS-tTA transgenes does not significantly damage learning during the training period.

Five GAL4 lines that had been shown previously to express in brain areas critical for CaMKII-dependent courtship conditioning (Joiner and Griffith, 1999) were crossed to the tetO-T287D; $U A S-t T A$ line and maintained until eclosion on tc food. Two lines, $30 Y$ and $M J 85 b$, showed significantly better training indices compared with the effector transgene controls (ANOVA; $F_{(6,127)}=6.7269 ;{ }^{\star} p<0.0001$, with planned post hoc comparisons significant; $\alpha=0.005$ ) (Fig. 3A). Locomotor defects did not account for the observed phenotypes (see Materials and Methods
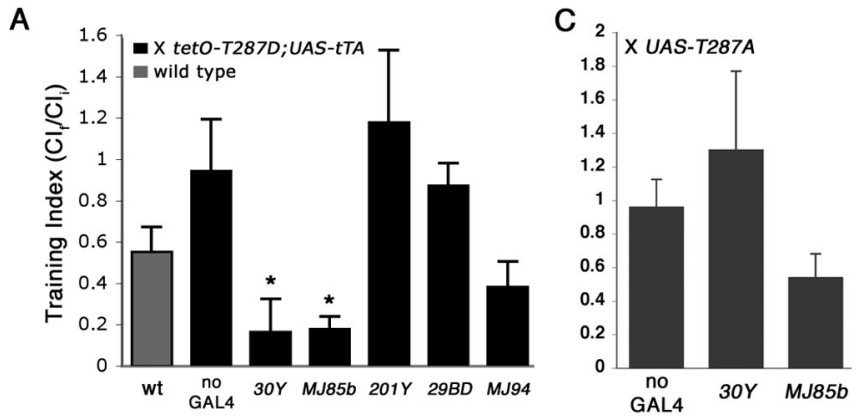

B
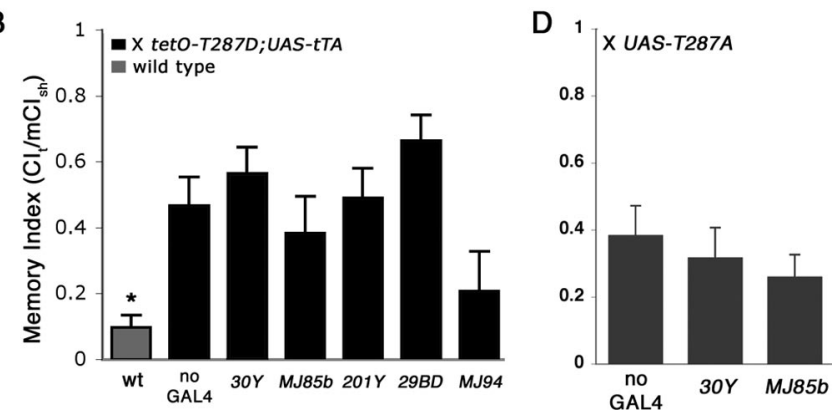

Figure 3. Courtship learning phenotypes of animals expressing T287D or T287A CaMKII in temporally and spatially restricted patterns. Animals expressing T287D or T287A CaMKII were trained for $1 \mathrm{hr}$ with a mated female and then tested immediately with an anesthetized virgin to assess memory. A, 30Y-and MJ85b-driven expression of T287D CaMKII improved training with the mated female. Gray bar shows wild-type (wt) (Canton S) performance. Black bars show training indices $\left(\mathrm{Cl}_{\mathrm{f}} / \mathrm{Cl}_{\mathrm{i}}\right)$ for the progeny of crosses between tet0-T287D; UAS-tTA and the GAL4 line indicated below the bar. 30Y- and MJ85b-driven expression of T287D significantly (ANOVA; $F_{(6,127)}=6.7269 ;{ }^{*} p<0.0001$, with planned post hoc comparisons significant; $\left.\alpha=0.005\right)$ enhanced suppression compared with the UAS-tTA; tet0-T287D effector control. All animals were raised on tetracycline until eclosion. $B$, T287D CaMKII does not affect associative memory. Gray bar shows wild-type (Canton S) performance. Black bars show memory indices $\left(\mathrm{Cl}_{\mathrm{t}} / \mathrm{mCl}_{\mathrm{sh}}\right)$ for the progeny of crosses between tet0-T287D; UAS-TTA and the GAL4 line indicated below the bar. None of the flies carrying a GAL4 insert performed significantly differently from the UAStTA/+; tet0-T287D/+ control (ANOVA; $F_{(6,124)}=6.3277 ; p<0.0001$, with planned post hoc comparisons not significant; $\alpha=0.005$ ). Wild-type flies performed significantly better than any animal carrying the effector transgenes (ANOVA; $F_{(6,124)}=6.3277 ;{ }^{*} p<0.0001$, with planned post hoc comparisons significant; $\alpha=0.005$ ). All flies were grown on tetracycline until eclosion. C, Expression of UAS-T287A CaMKII under control of 30Y or MJ85b did not affect performance during training compared with the UAS alone control (ANOVA; $F_{(2,44)}=1.5783 ; p>$ 0.2). All flies were grown on tetracycline-free medium. D, Expression of UAS-T287A CaMKII under control of $30 Y$ or MJ85b did not affect memory compared with the UAS alone control (ANOVA; $F_{(2.44)}=0.1734 ; p>0.8$ ). All flies were grown on tetracycline-free medium. For details of behavioral assays and statistics, see Materials and Methods.

and supplemental Table 1, available at www.jneurosci.org as supplemental material). The training enhancement was specific to the $\mathrm{Ca}^{2+}$-independent form of CaMKII. When $30 Y$ and MJ85b were used to overexpress a form of CaMKII that is not able to become $\mathrm{Ca}^{2+}$ independent (T287A CaMKII), performance during training did not differ from the effector transgene control $\left(\right.$ ANOVA; $F_{(2,44)}=1.5783 ; p=0.2178$ ) (Fig. $3 C$ ).

The striking $30 Y$ and $M J 85 b$ phenotypes required induction of T287D CaMKII expression during adulthood and were not the consequence of leaky developmental expression of the transgene. Feeding tc during adulthood completely blocked the enhancement in training, returning training indices to wild-type levels (Fig. 4). These results suggest that, for plasticity during the training period of courtship conditioning, the amount of total $\mathrm{Ca}^{2+}$ dependent CaMKII is not important, but the level of $\mathrm{Ca}^{2+}$ independent CaMKII is critical. 


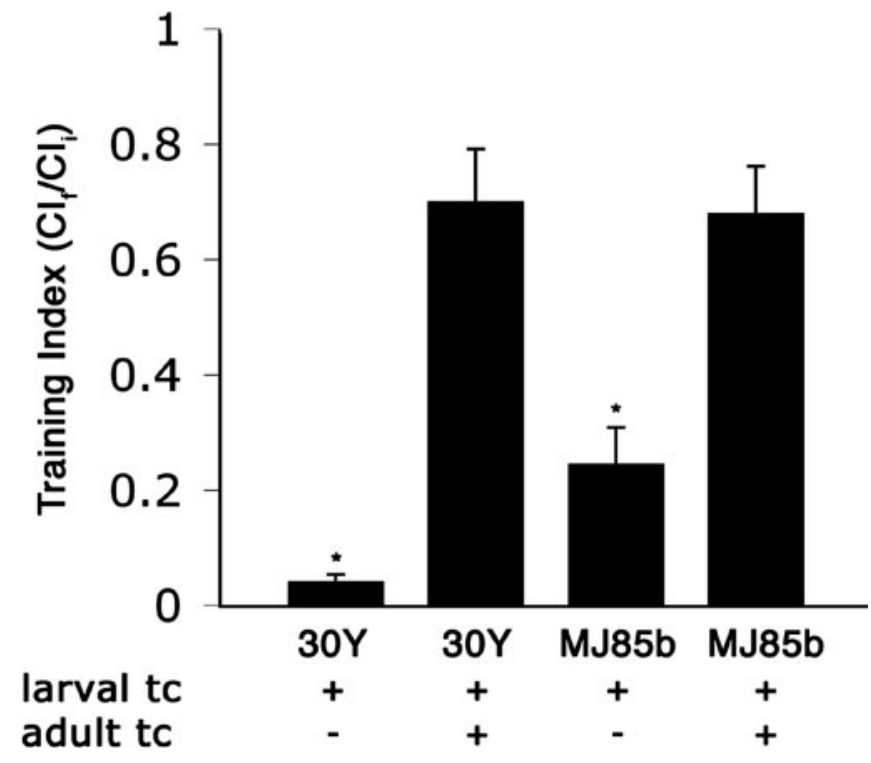

Figure 4. Training enhancement can be rescued by suppressing T287D CaMKII expression during adulthood. All flies were fed tc during larval life and then either treated with a sucrose carrier solution or a tc-sucrose solution $(100 \mu \mathrm{g} / \mathrm{ml})$ until testing. Feeding tet0-T287D/+; UAS-tTA/30Y and MJ85b; tet0-T287D/+; UAS-TTA/+ flies tc during adult life abolishes the enhanced training phenotype. Asterisks indicate that the tc-fed flies performed significantly better than the sucrose-fed adults for both $30 Y$ (ANOVA; $\left.F_{(1,38)}=38.1803 ; p<0.0001\right)$ and MJ85b (ANOVA; $\left.F_{(1,45)}=16.9041 ; p<0.0002\right)$.

\section{T287D CaMKII expression does not affect} short-term memory

For each genotype, memory was measured as the CI during a 10 min test period $\left(\mathrm{CI}_{t}\right)$ with a virgin female immediately after training over the mean sham control $\mathrm{CI}\left(\mathrm{mCI}_{\mathrm{sh}}\right)$. A lower memory index (Joiner and Griffith, 1997, 1999, 2000) indicates better short-term memory. Wild-type males raised on tc medium showed an exceptionally good associative memory score of $0.10 \pm 0.04$ compared with wild-type raised on tc-free food, which yielded a memory score of $0.29 \pm 0.09\left(t\right.$ test; $P_{(1,41)}=$ $0.0234)$. In the memory test, effector transgene control tet $O-T 287 \mathrm{D} /+$; UAS-tTA/+ males performed similarly to wild type raised on tc-free medium $\left(t\right.$ test; $\left.P_{(1,36)}=0.6783\right)$ but worse than tc-fed wild-type males $\left(t\right.$ test; $\left.P_{(1,39)}=0.0004\right)$. Not one of the five GAL4 lines we tested showed a significantly different memory score from the effector transgene control when driving the tet $O-T 287 D$ transgene $\left(\right.$ ANOVA; $F_{(6,124)}=$ 6.3277; $p<0.0001$, with planned post hoc comparisons not significant; $\alpha=0.005$ ) (Fig. $3 B$ ). Flies overexpressing the exclusively $\mathrm{Ca}^{2+}$-dependent T287A CaMKII also showed normal memory scores compared with the effecter control $\left(\right.$ ANOVA; $\mathrm{F}_{(2,44)}=0.1734 ; p=0.8413$ ) (Fig. $3 D$ ).

These results were somewhat surprising given previous work in which expression of a CaMKII-specific inhibitor peptide using these same GAL4 lines showed that CaMKII activity in mushroom bodies, central complex, and a subset of lateral protocerebral neurons was required for memory formation (Joiner and Griffith, 1999). Our data suggest that the absolute level of constitutive CaMKII activity is not the mediator of plasticity for memory formation in this assay. Endogenous, $\mathrm{Ca}^{2+}$-stimulable CaMKII is apparently both necessary and sufficient to form and express memory in this neural circuit.

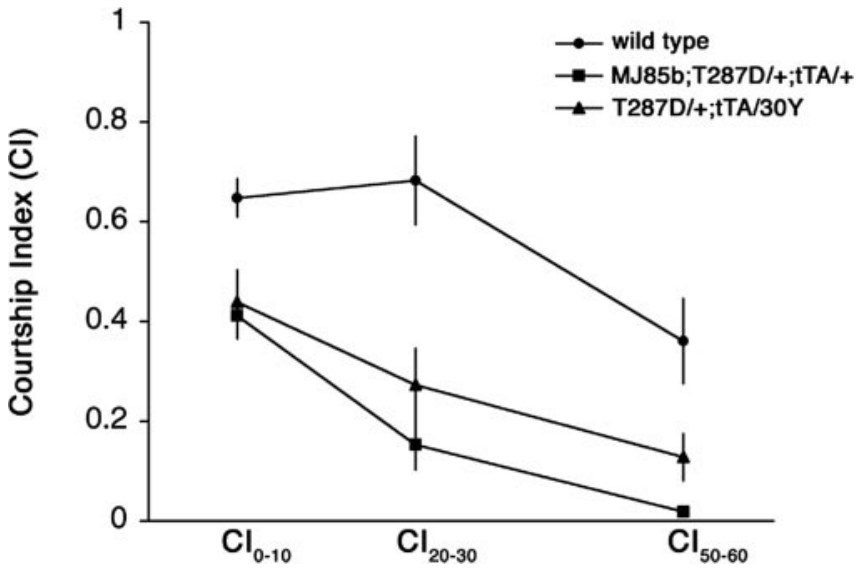

Figure 5. T287D CaMKII abolishes the lag to courtship suppression during training. Cls are shown for the initial $\left(\mathrm{Cl}_{0-10}, 0-10 \mathrm{~min}\right)$, middle $\left(\mathrm{Cl}_{20-30}, 20-30 \mathrm{~min}\right)$, and final $\left(\mathrm{Cl}_{50-60}\right.$, $50-60 \mathrm{~min}$ ) portions of the training phase. Halfway through training, wild-type males have not begun to suppress courtship. tet0-T287D/+; UAS-tTA/30Y and MJ85b; tet0-T287D/+; UAS-tTA/+ males start at a lower courtship level and are able to immediately begin to further suppress courtship.

\section{Constitutively active CaMKII defines a plasticity threshold for courtship suppression during training}

The data shown in Figure $3 A$ indicate that, at the end of the $1 \mathrm{hr}$ training period, there is a more profound suppression of courtship in males expressing T287D CaMKII under control of $30 Y$ and $M J 85 b$ when compared with the effecter control or T287D expressed with other GAL4 drivers. To determine whether this enhancement reflected a change in the dynamics of development of courtship suppression, we measured CIs at different times during the training period. Figure 5 shows that, for wild-type animals, suppression of courtship during training takes substantial time to develop. The level of courtship in the first $10 \mathrm{~min}$ of training is not significantly different from that measured between 20 and $30 \mathrm{~min}$ into the observation period. It is only by the last 10 min of the $1 \mathrm{hr}$ training period that there is a noticeable decrease in the level of courtship toward the trainer. In contrast, males that express T287D CaMKII start courting at a lower level and show progressively lower courtship throughout the training period.

Several important conclusions can be drawn from the time course data. First, T287D CaMKII males have a lower initial CI. This difference is T287D dependent. If $30 Y$ and MJ85b males are fed tc during adulthood, their CI for the first 10 min of the training period is not significantly different from control $(0.71 \pm 0.04$ and $0.58 \pm 0.05$; ANOVA; $F_{(5,117)}=1.4416 ; p=0.2145$ compared with control males). This suggests that males are "primed" to court less by the presence of the constitutively active CaMKII. Second, the slope of the relationship of CI to time over the course of training for T287D males is not significantly different from the slope for control males after the first 30 min of training (ANOVA; $\left.F_{(2,41)}=0.8054 ; p=0.4539\right)$. This suggests that courtship suppression occurs at the same rate in the $30 Y$ - and MJ85b-driven T287D males and wild-type control males but that control males have a threshold requirement for some factor before they can begin to suppress courtship behavior. Our data suggest that constitutively active CaMKII is the factor that builds up over time to permit courtship suppression.

\section{T287D CaMKII does not affect habituation to young males} Mature males will court immature males enthusiastically, but, over time during training, courtship decreases, and if subse- 
quently presented with a young male, the trained male will not court. This experience-dependent courtship modification has been shown to be habituation, which is a non-associative form of learning (Gailey et al., 1982). In contrast, memory of courtship conditioning after training with a mated female is known to be formed by an associative mechanism (Tompkins et al., 1983). The suppression of courtship during mated female training, however, is not as well understood at the mechanistic level. We reasoned that, if this suppression is a form of habituation, perhaps expression of T287D CaMKII would have a generally enhancing effect on courtship-related habituation. To test this, we examined the ability of $30 Y$ driving $\mathrm{Ca}^{2+}$-independent CaMKII to affect the response to immature males. We found that $30 Y$; tetO-T287D/+; UAS-tTA/+ males do not suppress courtship during training more effectively than the effector transgene tetO-T287D/+; UAS-tTA/+ controls [training indices $\left(\mathrm{CI}_{\mathrm{f}} / \mathrm{CI}_{\mathrm{i}}\right) 0.61 \pm$ 0.26 and $0.78 \pm 0.10 ; t$ test; $P_{(1,38)}=$ $0.9581 ; \alpha=0.05$ ]. In addition, males of both genotypes are able to suppress courtship of a subsequently presented immature male tester [memory index $\left(\mathrm{CI}_{\mathrm{t}} / \mathrm{mCI}_{\mathrm{con}}\right)$ for $30 Y$ driving $\mathrm{T} 287 \mathrm{D}$ CaMKII was $0.53 \pm 0.19$ compared with the effector transgene control, $0.59 \pm$ $0.11 ; t$ test; $\left.P_{(1,38)}=0.3059 ; \alpha=0.05\right]$. There are two possible interpretations of these data: either the action of $\mathrm{Ca}^{2+}$ independent CaMKII is specific to habituation toward mated females or the suppression of courtship during the training phase of the mated female courtship conditioning assay is not habituation. Our data do not allow us to distinguish between these two possibilities.

\section{Anatomical characterization of GAL4 expression patterns}

Correlation of the adult pattern of expression of the GAL4 lines used in this study with the behavioral phenotype obtained should allow us to identify candidate brain areas that are sensitive to levels of constitutively active CaMKII. The adult brain expression of all five of the lines used in this study has been described previously, but we reexamined them here (Fig. 6) with better resolution using $m C D 8-G F P$ to visualize all of the cell processes (Lee and Luo, 1999). MJ85b-GAL4 expresses throughout the PNS and CNS (Joiner and Griffith, 1997) (Fig. 6B). 29BD-GAL4 expresses in the $\alpha-, \beta$-, and $\gamma$-lobes of the mushroom bodies, as well as groups of
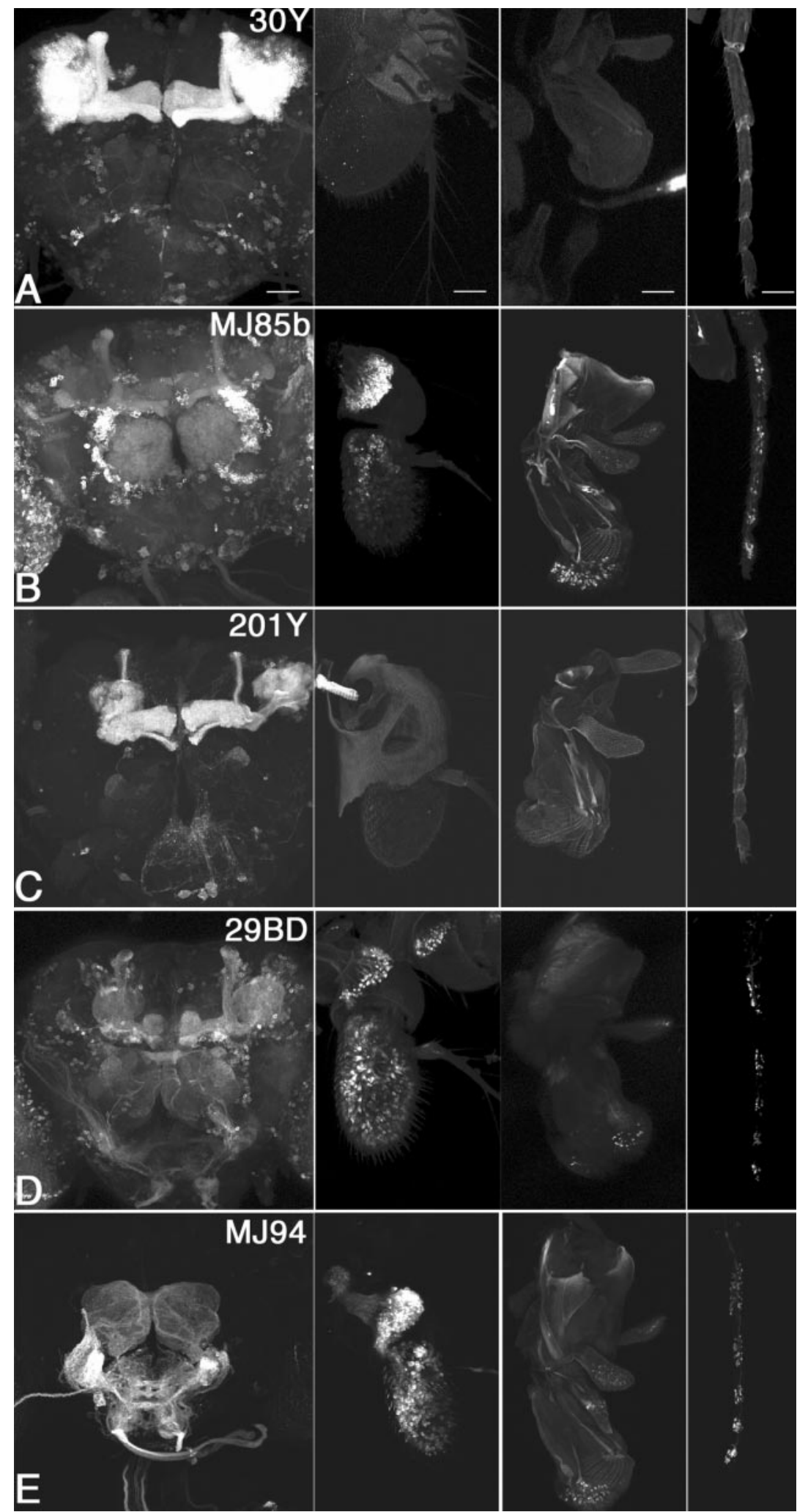

Figure 6. GAL4 expression patterns. GAL4-driven expression of UAS-mCD8GFP in adult male brains (first column), antennae (second column), proboscises (third column), and foretarsi (fourth right). $A, 30 Y$ expresses in mushroom bodies, antennal lobes, lateral protocerebrum, subesophageal ganglion, and optic lobes but not in the chemosensory organs. B, MJ85b expresses throughout the brain, as well as the antenna, proboscis, and foreleg. C, 201Y expresses primarily in the mushroom body, with expression in a few cells of the subesophageal ganglion, antennal lobe, and lateral protocerebrum, and no expression in chemosensory organs. D, 29BD expresses throughout the brain and chemosensory organs. $E$, MJ94 expresses in the subesophageal ganglion, with innervations of the antennal lobe, and in the antenna, proboscis, and foreleg. Images were collected as z-stacks on a Leica TCS SP2 confocal scanning microscope. Gain settings were not matched between genotypes. Scale bars: first and second columns, $40 \mu \mathrm{m}$; third and fourth columns, $80 \mu \mathrm{m}$. 
cells in the mediolateral protocerebrum and dorsolateral subesophageal ganglion (Joiner and Griffith, 1999) (Fig. 6D). The GAL4 line $30 Y$ expresses in the adult brain in the $\alpha-, \beta$-, and $\gamma$-lobes of the mushroom bodies, in cells of the medial lateral protocerebrum, cells of the dorsolateral subesophageal ganglion, "extrinsic" cells around the mushroom body, and antennal lobes (Yang et al., 1995; Joiner and Griffith, 1999) (Fig. 6A). GAL4 line $201 Y$ expresses throughout the $\gamma$-lobes, in the core of the $\alpha$ - and $\beta$-lobes, in cells of the pars intercerebralis, lateral protocerebrum, and subesophageal ganglion (Yang et al., 1995; Joiner and Griffith, 1999) (Fig. 6C). MJ94 expresses primarily in subesophageal ganglion, with afferents to the antennal lobes (Joiner and Griffith, 1999) (Fig. 6E).

In addition to looking at the CNS expression of these lines at higher resolution, we also examined the expression of these GAL4 lines in primary chemosensory organs. Expression of transgenes in these cells could have important consequences for information processing in courtship because this behavior is primarily driven by chemosensory (olfactory and gustatory) cues (Markow, 1987; Bray and Amrein, 2003). Figure 6 shows that MJ85b, 29BD, and MJ94 express in the antennae, proboscis (including labelum and maxillary palps), and forelegs of males (Fig. $6 B, D, E$ ), whereas $30 Y$ and $201 Y$ do not (Fig. 6A,C). Antennal and maxillary palp expression likely represents primary olfactory neurons, whereas expression in labelum and male foreleg is probably attributable to labeling of gustatory neurons (Stocker, 1994).

Correlation of the behavioral effects of T287D CaMKII with the spatial expression of these GAL4 lines suggests several conclusions. First, neither the absolute level of $\mathrm{Ca}^{2+}$-dependent CaMKII nor the level of constitutively active CaMKII is important for memory formation in this courtship conditioning paradigm. CaMKII activity per se is required (Griffith et al., 1993; Joiner and Griffith, 1999), but increasing $\mathrm{Ca}^{2+}$-dependent (T287A) or $\mathrm{Ca}^{2+}$-independent (T287D) CaMKII levels in mushroom bodies $(201 Y, 29 B D, 30 Y, M J 85 b)$, subsets of the lateral protocerebrum (30Y, MJ85b, 29BD, 201Y), subesophageal ganglion afferents to the antennal lobe (MJ94), or antennal lobe (30Y, MJ85b) neither blocked nor enhanced memory formation. Endogenous levels of CaMKII are necessary and sufficient to mediate memory formation.

The second conclusion that is suggested by the anatomical data are that the effect of T287D CaMKII during training is not mediated through either primary sensory neurons or intrinsic mushroom body neurons. Both $30 Y$ and MJ85b have strong expression in mushroom bodies, but $201 Y$ and $29 B D$ also express strongly in the mushroom bodies and do not give a training phenotype. The involvement of primary sensory neurons is ruled out by the fact that $30 Y$ has no expression in these areas. In addition, MJ94, which expresses strongly in all sensory structures, did not show an enhancement.

The major areas common to $30 Y$ and $M J 85 b$ expression patterns that are not found in the other lines are parts of the antennal lobe and a set of neurons extrinsic to the mushroom bodies. This is consistent with the requirement for CaMKII activity for normal training plasticity as assessed by expression of the ala CaMKII inhibitor peptide in antennal lobes and their afferents (Joiner and Griffith, 1999). This area of the brain is believed to be involved in initial processing of chemosensory information, and our results suggest that T287D CaMKII enhances this processing.

\section{Discussion}

\section{Calcium-independent CaMKII enhances courtship plasticity during training}

We observed an increase in the magnitude of courtship suppression during training with mated females when $30 Y$ or MJ85b drive expression of constitutively active CaMKII. The kinetics of suppression are also changed by T287D; the normal lag before suppression begins is abolished. These data suggest that, in the normal animal, constitutively active CaMKII must build up to a critical level before suppression of courtship of the mated female trainer can begin. Expression of T287D CaMKII boosts the basal level of $\mathrm{Ca}^{2+}$-independent activity and pushes the circuit over threshold, allowing immediate suppression of courtship behavior. Previous studies in rodents have shown that constitutively active CaMKII can alter or enhance long-term depression and long-term potentiation (LTP) (Lledo et al., 1995; Mayford et al., 1995; Bejar et al., 2002), but, in all cases in which a whole animal behavioral output was measured, constitutive CaMKII activity disrupted learning (Mayford et al., 1996; Bejar et al., 2002). Our study is the first report of the activated form of CaMKII enhancing a behavioral output.

The enhancement of courtship suppression was specific to a particular population of CNS neurons. GAL4 lines with other expression patterns (MJ94, 201Y, and 29BD) did not show this effect. The regions of expression in $30 Y$ that are not present in these other lines include extrinsic cells surrounding the mushroom bodies and cells of the antennal lobes. It is possible that these neurons innervate the mushroom bodies and could lie either upstream or downstream of associative structures in the courtship-suppression circuitry. Investigation into the identity of and connections made by these extrinsic neurons may provide insight into the processing of mushroom body-bound information.

\section{Mechanisms of T287D plasticity enhancement}

The cellular mechanism of action of constitutive CaMKII in this population of cells is threshold based. Rodent physiology findings suggest that, in hippocampus, the amount of constitutively active CaMKII is important for setting the threshold of frequency response for LTP. Using tetracycline-controlled expression of T286D $\alpha$ CaMKII in mice, Bejar et al. (2002) showed that these effects were dose dependent and that low levels of kinase produced an enhancement of low-frequency LTP, whereas high levels blocked stimulation of LTP by low-frequency stimulation. This implies that the effects of constitutive CaMKII on behavioral output are likely to be dose dependent and that high doses may lead to behavioral defects by saturating the plasticity process. This is consistent with our finding that there appears to be a threshold effect for courtship suppression. Additional support for this idea comes from expression of T287D CaMKII under control of the strong antennal lobe GAL4 line GH146 (Heimbeck et al., 2001), which produces animals that fail to court (J. E. Mehren, unpublished results). In this case, the threshold level of constitutive kinase may be exceeded and courtship is fully suppressed. In $30 Y$ animals, which also express in some antennal lobe cells, initial courtship is lower than wild type, but there is room for further suppression. The difference between these lines may not only be attributable to the number of cells affected but the dose of T287D delivered to each cell.

At the biochemical level, how does constitutively active CaMKII function differently from the $\mathrm{Ca}^{2+}$-dependent form of the kinase? An early idea about the role of constitutive kinase activity was that it might simply prolong the lifetime of the active kinase, allowing it to keep its important substrates phosphory- 
lated for a longer period after the initial stimulus. Our increasingly sophisticated understanding of CaMKII localization and biochemistry has necessitated revision of this model. Activation of CaMKII by autophosphorylation can allow it to bind to neuronal scaffolding proteins, which will affect the array of substrates to which the kinase has access (for review, see Griffith et al., 2003). Autophosphorylation of T286 rat $\alpha$ CaMKII also affects the dynamics of CaMKII translocation to and from synaptic sites (Shen and Meyer, 1999). In addition, the fact that T287D CaMKII is active at low levels of cellular calcium may allow it access to substrates whose phosphorylation is blocked by $\mathrm{Ca}^{2+}$ dependent interactions with other proteins (Hashimoto et al., 1988). Differential localization and substrate access therefore may be responsible for the unique effects on neuronal function seen with T287A and T287D CaMKII (Park et al., 2002; this study).

\section{Tissue-specific roles of different CaMKII activity states}

The ability of T287D CaMKII, when expressed in a subset of CNS neurons, to enhance plasticity during the training period of courtship conditioning is interesting, but the failure of constitutive CaMKII to affect memory formation (either positively or negatively) when expressed in the mushroom bodies is equally interesting. In previous studies, when UAS-ala was used to investigate the requirement for CaMKII activity, all of the mushroom body-expressing GAL4 lines used in the current study showed defective memory (Joiner and Griffith, 1999, 2000). The ala transgene encodes a CaMKII inhibitor peptide that should block both $\mathrm{Ca}^{2+}$-dependent and $\mathrm{Ca}^{2+}$-independent CaMKII. When the same GAL4 lines drove constitutively active CaMKII in the current study, there was no decrease in memory formation. These data suggest that the mechanisms by which CaMKII facilitates plasticity differ in a neuron-specific manner.

Our findings suggest that some cells can require CaMKII activity for plasticity but be unaffected by additional constitutive CaMKII. Several functional aspects of CaMKII can be manipulated by expression of transgenes: total activity can be decreased (ala peptide), $\mathrm{Ca}^{2+}$-dependent activity can be increased (T287A CaMKII), and $\mathrm{Ca}^{2+}$-independent activity can be increased (T287D CaMKII). The effects of each of these manipulations will depend on the details of the mechanism by which CaMKII regulates memory formation in that neuron type. Memory defects with expression of ala peptide suggest that some form of CaMKII activity is required. If CaMKII function is important for plasticity during epochs of high calcium, expression of T287A and T287D could have similar phenotypic effects. If the role of CaMKII in that particular cell requires generation of the constitutive form of the kinase, T287D, but not T287A, might produce a phenotype. The important variable as to whether a phenotype will be produced is the nature of the CaMKII-dependent biochemistry in that neuron; only processes that are saturable or threshold-based are likely to be disrupted by overexpression. For this first type of neuron, addition of more $\mathrm{Ca}^{2+}$-independent CaMKII could either push the process closer to threshold and enhance learning or it could push it past threshold and prevent modulation. This study revealed a CaMKII threshold requirement in specific cells of the Drosophila CNS for courtship suppression to occur during training. Neurons that use biochemical events that rely on relative changes of phosphorylation will be insensitive if they are capable of operating from a range of baseline states. The unchanged memory of mutants overexpressing constitutively active CaMKII in this study reveals neurons of this second type in the Drosophila brain.
Our results, and the results of experiments in mice, suggest that both saturable and nonsaturable CaMKII-dependent mechanisms are used for memory formation in vivo. In rodent hippocampus, CaMKII activity is required for learning in the Morris water maze (Silva et al., 1996), and overexpression of T286D $\alpha$ CaMKII blocks learning (Mayford et al., 1996), suggesting a saturable CaMKII-dependent process. In habituation of Drosophila leg flexion, CaMKII activity is required, and overexpression of either the $\mathrm{Ca}^{2+}$-dependent or $\mathrm{Ca}^{2+}$-independent form disrupts plasticity but in qualitatively different manners (Jin et al., 1998). In rodent barrel cortex, CaMKII activity is required for vibrissa deprivation plasticity (Glazewski et al., 1996), but overexpression of constitutively active CaMKII does not enhance or block plasticity (Glazewski et al., 2001), suggesting a nonsaturable CaMKII-dependent process. In Drosophila courtship conditioning, we were able to test a number of different neuron groups and sub-behaviors in parallel. CaMKII activity is required for immediate memory, but overexpression of neither $\mathrm{Ca}^{2+}$ dependent nor $\mathrm{Ca}^{2+}$-independent CaMKII has an effect on the process (Joiner and Griffith, 1999; this study). CaMKII is also required for modulation of courtship during training, and, in this behavior, addition of more $\mathrm{Ca}^{2+}$-independent kinase facilitates the process.

In all of these experiments, constitutive CaMKII was provided to cells that still had normal levels of endogenous kinase. In the case of hippocampal learning, there is evidence from cellular assays that overexpression of $\mathrm{Ca}^{2+}$-independent CaMKII can saturate the plasticity machinery (Lledo et al., 1995; Bejar et al., 2002). In the case of rodent barrel cortex and courtship memory formation, endogenous kinase is apparently sufficient even in the face of elevated basal $\mathrm{Ca}^{2+}$-independent activity to perform the critical CaMKII-dependent functions in those neurons. The failure of excess T286D CaMKII to disrupt plasticity suggests that, in these cortical cells and in the mushroom bodies, there is no saturable process that overexpression of the constitutive form of the kinase can occlude. Interestingly, the mechanism by which the endogenous kinase functions in barrel cortex does apparently include a requirement for $\mathrm{Ca}^{2+}$-independent CaMKII activity because, if the endogenous gene is replaced with a point mutant (T286A) that cannot become $\mathrm{Ca}^{2+}$ independent, barrel plasticity is blocked (Glazewski et al., 2000). Clearly the cellular and molecular mechanisms that subserve CaMKII-dependent plasticity represent a rich variety of processes. Manipulations of total CaMKII activity and the levels of $\mathrm{Ca}^{2+}$-dependent and $\mathrm{Ca}^{2+}$ independent forms of CaMKII can be used to tease apart the roles and mechanisms of this ubiquitous protein in memory formation.

\section{References}

Bach ME, Hawkins RD, Osman M, Kandel ER, Mayford M (1995) Impairment of spatial but not contextual memory in CaMKII mutant mice with a selective loss of hippocampal LTP in the range of the theta frequency. Cell 81:905-915.

Bejar R, Yasuda R, Krugers H, Hood K, Mayford M (2002) Transgenic calmodulin-dependent protein kinase II activation: dose-dependent effects on synaptic plasticity, learning, and memory. J Neurosci 22:5719-5726

Bello B, Resendez-Perez D, Gehring WJ (1998) Spatial and temporal targeting of gene expression in Drosophila by means of a tetracycline-dependent transactivator system. Development 125:2193-2202.

Blau HM, Rossi FM (1999) Tet B or not tet B: advances in tetracyclineinducible gene expression. Proc Natl Acad Sci USA 96:797-799.

Brand AH, Dormand EL (1995) The GAL4 system as a tool for unraveling the mysteries of the Drosophila nervous system. Curr Opin Neurobiol 5:572-578.

Bray S, Amrein H (2003) A putative Drosophila pheromone receptor ex- 
pressed in male-specific taste neurons is required for efficient courtship. Neuron 39:1019-1029.

Fong YL, Taylor WL, Means AR, Soderling TR (1989) Studies of the regulatory mechanism of $\mathrm{Ca}^{2+} /$ calmodulin-dependent protein kinase II. Mutation of threonine 286 to alanine and aspartate. J Biol Chem 264:16759-16763.

Gailey DA, Jackson FR, Siegel RW (1982) Male courtship in Drosophila: the conditioned response to immature males and its genetic control. Genetics 102:771-782.

Glazewski S, Chen CM, Silva A, Fox K (1996) Requirement for alphaCaMKII in experience-dependent plasticity of the barrel cortex. Science 272:421-423.

Glazewski S, Giese KP, Silva A, Fox K (2000) The role of alpha-CaMKII autophosphorylation in neocortical experience-dependent plasticity. Nat Neurosci 3:911-918.

Glazewski S, Bejar R, Mayford M, Fox K (2001) The effect of autonomous alpha-CaMKII expression on sensory responses and experiencedependent plasticity in mouse barrel cortex. Neuropharmacology 41:771-778.

Greenspan RJ (1995) Understanding the genetic construction of behavior. Sci Am 272:72-78.

Griffith LC, Verselis LM, Aitken KM, Kyriacou CP, Greenspan RJ (1993) Inhibition of calcium/calmodulin-dependent protein kinase in Drosophila disrupts behavioral plasticity. Neuron 10:501-509.

Griffith LC, Lu CS, Sun XX (2003) CaMKII, an enzyme on the move: regulation of temporospatial localization. Mol Interv 3:386-403.

Hall JC (2002) Courtship lite: a personal history of reproductive behavioral neurogenetics in Drosophila. J Neurogenet 16:135-163.

Hashimoto Y, King MM, Soderling TR (1988) Regulatory interactions of calmodulin-binding proteins: phosphorylation of calcineurin by autophosphorylated $\mathrm{Ca}^{2+} /$ calmodulin-dependent protein kinase II. Proc Natl Acad Sci USA 85:7001-7005.

Heimbeck G, Bugnon V, Gendre N, Keller A, Stocker RF (2001) A central neural circuit for experience-independent olfactory and courtship behavior in Drosophila melanogaster. Proc Natl Acad Sci USA 98:15336-15341.

Hudmon A, Schulman H (2002) Structure-function of the multifunctional $\mathrm{Ca}^{2+} /$ calmodulin-dependent protein kinase II. Biochem J 364:593-611.

Jin P, Griffith LC, Murphey RK (1998) Presynaptic $\mathrm{Ca}^{2+} /$ calmodulindependent protein kinase II regulates habituation of a simple reflex in adult Drosophila. J Neurosci 18:8955-8964.

Joiner MA, Griffith LC (1997) CaM kinase II and visual input modulate memory formation in the neuronal circuit controlling courtship conditioning. J Neurosci 17:9384-9391.

Joiner MA, Griffith LC (1999) Mapping of the anatomical circuit of CaM kinase-dependent courtship conditioning in Drosophila. Learn Mem 6:177-192.

Joiner MA, Griffith LC (2000) Visual input regulates circuit configuration in courtship conditioning of Drosophila melanogaster. Learn Mem $7: 32-42$.

Kane NS, Robichon A, Dickinson JA, Greenspan RJ (1997) Learning without performance in PKC-deficient Drosophila. Neuron 18:307-314.

Lee T, Luo L (1999) Mosaic analysis with a repressible cell marker for studies of gene function in neuronal morphogenesis. Neuron 22:451-461.
Lin DM, Goodman CS (1994) Ectopic and increased expression of Fasciclin II alters motoneuron growth cone guidance. Neuron 13:507-523.

Lin DM, Fetter RD, Kopczynski C, Grenningloh G, Goodman CS (1994) Genetic analysis of Fasciclin II in Drosophila: defasciculation, refasciculation, and altered fasciculation. Neuron 13:1055-1069.

Lisman J, Schulman H, Cline H (2002) The molecular basis of CaMKII function in synaptic and behavioural memory. Nat Rev Neurosci 3:175-190.

Lledo PM, Hjelmstad GO, Mukherji S, Soderling TR, Malenka RC, Nicoll RA (1995) Calcium/calmodulin-dependent kinase II and long-term potentiation enhance synaptic transmission by the same mechanism. Proc Natl Acad Sci USA 92:11175-11179.

Markow TA (1987) Behavioral and sensory basis of courtship success in Drosophila melanogaster. Proc Natl Acad Sci USA 84:6200-6204.

Mayford M, Wang J, Kandel ER, O’Dell TJ (1995) CaMKII regulates the frequency-response function of hippocampal synapses for the production of both LTD and LTP. Cell 81:891-904.

Mayford M, Bach ME, Huang YY, Wang L, Hawkins RD, Kandel ER (1996) Control of memory formation through regulated expression of a CaMKII transgene. Science 274:1678-1683.

Park D, Coleman MJ, Hodge JJL, Budnik V, Griffith LC (2002) Regulation of neuronal excitability in Drosophila by constitutively active CaMKII. J Neurobiol 52:24-42.

Shen K, Meyer T (1999) Dynamic control of CaMKII translocation and localization in hippocampal neurons by NMDA receptor stimulation. Science 284:162-166.

Siegel RW, Hall JC (1979) Conditioned responses in courtship behavior of normal and mutant Drosophila. Proc Natl Acad Sci USA 76:565-578.

Silva AJ, Rosahl TW, Chapman PF, Marowitz Z, Friedman E, Frankland PW, Cestari V, Cioffi D, Sudhof TC, Bourtchuladze R (1996) Impaired learning in mice with abnormal short-lived plasticity. Curr Biol 6:1509-1518.

Sokal RR, Rohlf HR (1995) Biometry, Ed 3. New York: Freeman.

Stocker RF (1994) The organization of the chemosensory system in Drosophila melanogaster: a review, p 239. Cell Tissue Res 275:3-26.

Tompkins L, Siegel RW, Gailey DA, Hall JC (1983) Conditioned courtship in Drosophila and its mediation by association of chemical cues. Behav Genet 13:565-578.

Villella A, Hall J (1996) Courtship anomalies caused by doublesex mutations in Drosophila melanogaster. Genetics 143:331-344.

Villella A, Gailey DA, Berwald B, Ohshima S, Barnes PT, Hall JC (1997) Extended reproductive roles of the fruitless gene in Drosophila melanogaster revealed by behavioral analysis of new fru mutants. Genetics 147:1107-1130.

Waldmann R, Hanson PI, Schulman H (1990) Multifunctional $\mathrm{Ca}^{2+} /$ calmodulin-dependent protein kinase made $\mathrm{Ca}^{2+}$ independent for functional studies. Biochemistry 29:1679-1684.

Wang Z, Palmer G, Griffith LC (1998) Regulation of Drosophila $\mathrm{Ca}^{2+}$ / calmodulin-dependent protein kinase II by autophosphorylation analyzed by site-directed mutagenesis. J Neurochem 71:378-387.

Yang MY, Armstrong JD, Vilinsky I, Strausfeld NJ, Kaiser K (1995) Subdivision of the Drosophila mushroom bodies by enhancer-trap expression patterns. Neuron 15:45-54. 